DOE/ER/ $14004--3$

DE92 010795

\title{
THE MOLECULAR CHARACTERIZATION
}

\author{
OF THE
}

LIGNIN-FORMING PEROXIDASE

DE-FG02-89ER14004

\section{PROGRESS SUMMARY REPORT}

\author{
(April 1, 1989 - March 31, 1992) \\ L. Mark Lagrimini \\ Department of Horticulture \\ The Ohi State University \\ 2001 Fyffe Court \\ Columbus, Ohio 43210-1096
}

\section{DISCLAIMER}

This report was prepared as an account of work sponsored by an agency of the United States Government. Neither the United States Government nor any agency thereof, nor any of their employees, makes any warranty, express or implied, or assumes any legal liability or responsibility for the accuracy, sompleteness, or usefulness of any information, apparatus, product, or process disclosed, or represents that its use would not infringe privately owned rights. Reference herein to any specific commercial product, process, or service by trade name, trademark, manufacturer, or otherwise does not necessarily constitute or imply its endorsement, recommendation, or favoring by the United States Government or any agency thereof. The views and opinions of authors expressed herein do not necessarily state or reflect those of the United States Government or any agency thereof. 


\section{PROGRESS REPORT}

This laboratory is committed to understanding the function of plant peroxidases via a multi-disciplinary approach. We have chosen the lignin-forming peroxidase frorn tobacco as the first isoenzyme to be subjected to this comprehensive approach. The goals which were set out upon the initiation of this project were as follows: (1) utilize a cDNA clone to the tobacco anionic peroxidase to generate transgenic plants which either over-produced this isoenzyme or specifically under-produced this isoenzyme via antisense RNA, (2) describe any phenotypic changes resulting from altered peroxidase expression, (3) perform morphological, physiological, and biochemical analysis of the above mentioned plants to help in determining the in planta function for this enzyme, and (4) clone and characterize the gene for the tobacco anionic peroxidase. A summary of progress thus far which includes both published and unpublished work will be presented in three sections: generation and characterization of transgenic plants, description of phenotypes, and biochemical and physiological analysis of peroxidase function, and cloning and characterization of the tobacco anionic peroxidase gene.

(1) Generation of transgenic plants with altered peroxidase activity.

A cDNA clone for the tobacco anionic peroxidase was joined to the intact CaMV 355 promoter. This configuration will provide high levels of expression of peroxidase in most tissues throughout the plant. This chimeric gene was inserted into a binary transformation vector ( $\mathrm{pCib} 10$ ) which includes a selectable kanamycin resistance gene. The resulting plasmid was transformed into Agrobacterium tumefaciens strain A136, which was co-cultivated with leaf tissue from $N$. tabacum and $N$. sylvestris plants (Lagrimini et al., 1990). Plants were regenerated on solid media containing kanamycin. A parallel experiment was conducted with the 5 ' half of the peroxidase CDNA joined in an antisense configuration with respect to the $35 S$ promoter (Rothstein and Lagrimini, 1989). This configuration will produce antisense RNA to the anionic peroxidase in most cell types, thus reducing the expression of the endogenous anionic peroxidase gene. Controls were performed with the pCib10 vector alone.

Several plants vich were regeneraied in the presence of kanamycin with either of the chimeric peroxidase genes were transferred to pots and grown in the greenhouse. When the plants were $40 \mathrm{~cm}$ tall, leaf tissue was assayed for peroxidase activity. It was found that peroxidase activity was more than tenfold greater in plants with the overproducer construct than in control plants. Less than one-tenth the peroxidase activity of control plants was found in transformed plants with the antisense RNA construct (Lagrimini et al., 1991). Plants were allowed to self-fertilize, and seed was collected when possible.

(2) Description of phenotypic changes as a result of altered peroxidase activity, and the morphological, physiological, and biochemical analysis of transformed plants.

Wilting phenotype. Transgenic plants which overproduced the arionic peroxidase 
by tenfold were phenotypically similar to wild-type plants until the plants flowered. Upon flowering the transformed plants began to wilt severely, respective of watering regime (Lagrimini et al., 1990). We initially determined that wilting was not a result of water loss through the leaf surface, nor was it a result of abnormal vessel morphology in the stem and leaf veins. Since these results were published, we have measured leaf water potential, rate of water uptake, and root hydraulic conductivity. We have also measured the growth rates of leaf and roots, and have performed graiting experiments between wild-type and peroxidase overproducer plants. Leaf water potential is significantly reduced $(-14.3 \pm 1.3 \mathrm{Bars})$ in a wilted leaf as compared to a turgid leaf from a control plant $(-3.08 \pm 1.2$ Bars). However, if a wilted leaf is allowed to recover turgidity by placing in a beaker of water under subdued lighting, water potential returns to near wild-type levels $(-4.33 \pm 2.0$ Bars $)$. This is an indication that the water relations of a leaf from a transformed plant is not significantly different than a control leaf.

The morphology and physiology of the stem and leaf from a peroxidase overproducer plant was so similar to that of a control plant that our attention was taken to the roots. We attempted to graft shoots from a peroxidase overproducer plant onto control root stock and visa versa. We were able to show that shoots from a peroxidase overproducer plant will not wilt when grafted onto root stock of a control plant. This reveals that peroxidase overproduction exerts its effect on water relations through the roots. We have not been able to successfully graft control shoots onto overproducer plants. This result can be explained by another

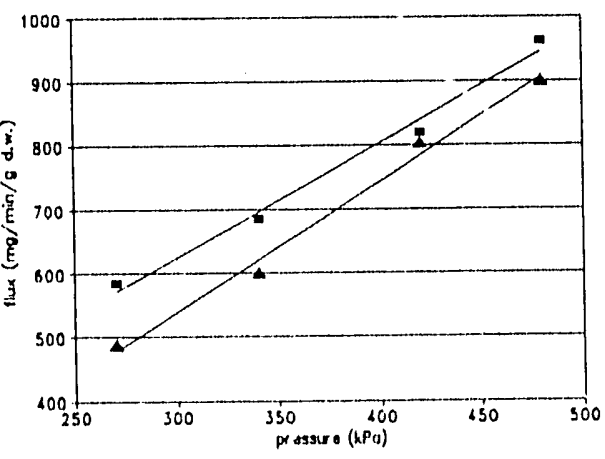

Figure 1. Root flux of control (m-- $\mathbf{m})$ and peroxidase overproducer plants (--). phenotype which be described later.

It became necessary to ascertain if the roots were defective in form or function. Root hydraulic conductivity was measured in the laboratory of Dr. Robert Joly at Purdue University. Intact roots from mature plants at anthesis were place in a pressure chamber, and flux was monitored at various pressures. Hydraulic conductivity of wild-type and transformed roots was found to be nearly identical (Fig. 1). This indicated that the roots from the peroxidase overproducer plant were functionally normal.

Infertility. When plants expressing antisense RNA, which had greater than a $90 \%$ reduction in peroxidase activity, were allowed to self-fertilize it was found that they were nearly infertile. These plants had a reduced fertility that exhibited seasonal variation, with an extremely low seed set $(<5 \%$ of control) during the summer months when greenhouse temperatures were high $\left(>34^{\circ} \mathrm{C}\right)$. Examination of the reproductive organs of plants with suppressed peroxidase activity revealed a $17 \%$ increase in the size of the stamen, stigma, style and receptacles at maturity. The pollen stained positive for viability with fluorescein diacetate; however, the frequency 
of in vitro pollen germination was reduced by as much as $75 \%$. Sexual crosses between wild-type and transformed tobaccp have also indicated male infertility.

During the winter months seed set recovered to near normal levels. However, when seed were germinated, it was found that fewer progeny than predicted were kanamycin resistant, and that even fewer progeny had reduced peroxidase activity. Apparently, the anionic peroxidase is required during embryo or seed development which is in addition to the male infertility described above. Initial kanamycin resistant regenerates $\left(R_{0}\right)$ with $>$ tenfold suppression of peroxidase activity were propagated through shoot regeneration media in the presence of kanamycin. Kanamycin resistant plants were obtained; however, these plants had wild-type peroxidase levels. Although shoot differentiation in cell culture is different than embryogenesis, the anionic peroxidase is evidently required, and we have selected for tissues which have averted the antisense RNA suppression. These phenomenon have greatly reduced the number of experiments which we could perform on antisense RNA plants, and have limited us to propagating $R_{0}$ plants asexually through shoot tips.

Browning phenotype. Transgenic plants which overproduce peroxidase also have a brown discolored cambium, and pith tissue browns rapidly after wounding. The browning can be attributed to the deposition of polyphenolic acids. This research was published in Lagrimini, $199 \hat{i}$. The purified anionic peroxidase was shown to catalyze the $\mathrm{H}_{2} \mathrm{O}_{2}$-dependent polymerization of caffeic and chlorogenic acid in vitro. These reactions exhibit modified ping-pong kinetics which prohibit the determination of a Michaelis constant. The levels of soluble phenols and polyphenol oxidase activity are the same in control and overproducer plants. Wound-induced lignification was found to occur in both control and overproducer pith tissue, however, lignification occurred 2 days sooner in overproducer plants. This indicates that peroxidase may be the limiting factor in stress- and disease-induced lignification.

Cell size and epinasty. One of the best examples of a continuum from peroxidase suppression through peroxidase overproduction is the effect on leaf cell size. Crosssections through leaf tissue from control and transformed plants have revealed cell size is inversely proportional to peroxidase activity. We found leaf cells to be $\approx 17 \%$ larger in plants with 20 -fold reduced peroxidase activity, and $\approx 30 \%$ smaller in peroxidase overproducer plants when compared to control plants (Lagrimini, submitted for publication).

There are three possible scenarios for how peroxidase could influence leaf cell size. First, peroxidase is thought to introduce cross-links into cell walls which creates a resistance to cell expansion. More or less peroxidase would influence the extent of cell wall elongation. Alternatively, peroxidase is modulating either water uptake or transport through the roots. We know that elevated peroxidase activity in root tissue results in decreased turgor pressure to the point of wilting. Turgor pressure along with cell wall expansion are required for growth. Finally, peroxidase may have a role in auxin metabolism which is known to control cell expansion.

In addition to increased leaf thickness, the transformad plants which underproduce peroxidase have leaves which were epinastic (Rothstein and Lagrimini, 1989; Lagrimini et al., submitted for publication). Epinasty and leaf distortion are 
known to be caused by ethylene or auxin-induced ethylene. This led us to believe that auxin or ethyione metabolism was affected.

Auxin catabolism. Many of the phenotypes described above such as epinasty, infertility, and alteration of cell size could be attributed to changes in auxin metabolism. Peroxidase is known to catalyze the oxygen-dependent decarboxylation of IAA in vitro; therefore, I sought out to determine if the phenotypes seen in transgenic plants were a result of peroxidases influence on auxin levels. These results are presented in a manuscript submitted for publication (Lagrimini, 1991). In summary, in vitro auxin oxidation rates were determined with purified anionic peroxidase and $1-\left[{ }^{14} \mathrm{C}\right]-\mid \mathrm{AA}$, in vivo oxidation rates were determined by infiltrating tissue with $1-\left[{ }^{14} \mathrm{C}\right]-\mid \mathrm{AA}$, and the steady-state free IAA levels were determined in leaf tissue from control and transformed plants by GC-MS.

The purified tobacco anionic peroxidase was assayed in vitro and determined to have a $\mathrm{Km}$ for IAA of $610 \mu \mathrm{M}$ with a Vmax of $8.99 \times 10^{-11}$ moles $\mathrm{IAA} / \mathrm{min}$. Leaf tissue was incubated with $\left[1-{ }^{14} \mathrm{C}\right] \mid \mathrm{AA}$ and the rate of ${ }^{14} \mathrm{CO}_{2}$ evolution was similar to the rate predicted for the purified tobacco anionic peroxidase based on the kinetic parameters determined in vitro. However, when the steady-state level of endogenous IAA in planta was determined in equivalent leaf tissue from control, twenty-fold peroxidase underproducer, and tenfold peroxidase overproducer plants no significant difference in free IAA was found. By calculation, the predicted rate of IAA decarboxylation by peroxidase in vivo would be only $4.95 \times 10^{-14} \mathrm{moles} / \mathrm{min}$ based on a concentration of $3.36 \times 10^{-7} \mathrm{M}$ IAA. This would result in at most a $0.9 \%$ loss of IAA for every $10 \mathrm{~cm}$ traveled through the plant.

It remains to be determined if auxin is metabolized by the anionic peroxidase. It is my hypothesis that the phenotypes which range from variations in cell size, to infertility, and from chronic wilting to epinasty could most easily be explained by the effect of a phytohormone. Auxin is the most reasonable candidate because of the known oxidative decarboxylation of IAA by peroxidase in vitro. This cannot exclude the possibility that peroxidase is directly creating these phenotypes. So little is known about the function of peroxidase in planta that the potential for altering a number of physiological processes does exist.

(3) Cloning and characterization of the lignin-forming peroxidase gene.

Isolation of genomic clones. Genomic DNA was isolated from N. tabacum leat tissue and digested with EcoR I (* activity). These fragments were ligated into the lambda phage vector EMBL3 digested with EcoR I. A tobacco genomic DNA library consisting of over 100,000 genomic fragments was constructed. This library was screened with the TobAnPOD CDNA, and three genomic clones were isolated. Restriction analysis revealed that two of these clones were identical ( $\lambda$ POD1 and $\lambda P O D 2)$. A second isolate, $\lambda P O D 3$ was unique. It was shown earlier that $N$. tabacum has four copies of the anionic peroxidase gene (Lagrimini et al., 1987). By comparative blots of $N$. tabacum, $N$. sylvestris, and $N$. tomentosiformis DNA it could be determined that all clones were originally derived from $N$. sylvestris. 
Sequencing of two genomic clones. The genomic clone $\lambda P O D 1$ was subcloned and sequenced first. Partial sequence of this clone revealed that this genomic DNA fragment actually contained a pseudogene for the tobacco anionic peroxidase (Fig. 2).

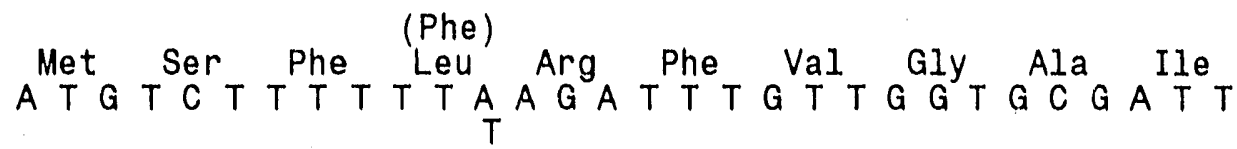

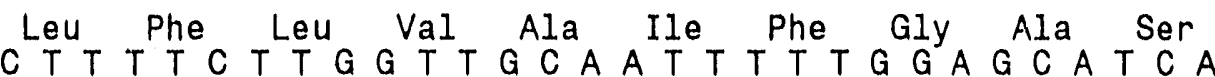

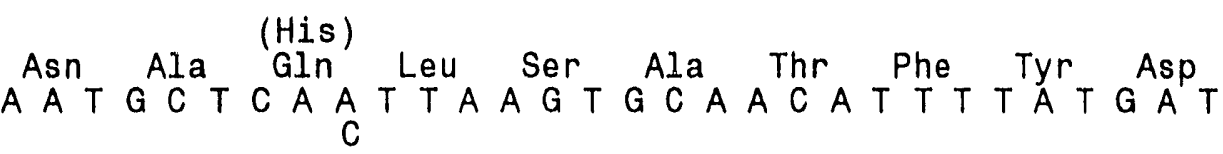

$$
\begin{aligned}
& \text { (Cys) Thr Cys Pro Asn Val Thr Ser Ile Val } \\
& A C C A C C T T G C C C T A A T G T T A C A A G T A T T G T A \text { T } T \text { (ins) } \\
& T G \text { Arg Gly Val Met }(\text { Asp }) \text { Gin Arg Gln Arg Thr } \\
& C G T G G T G T T A T G G A T C A A A G G C A A C G T A C T
\end{aligned}
$$

Figure 2. Partial sequence of the tobacco anionic peroxidase pseudogene, $\lambda P O D 1$. Amino acids in parentheses indicate changes from the cDNA. An insertion is also indicated (ins).

The high frequency of changes ( $1 \mathrm{bp}$ change per 50bp), the elimination of an absolutely conserved cysteine residue, and the insertion of a $T$ residue which disrupts the reading frame make us certain that this is a pseudogene and not a related peroxidase gene or some other artifact.

The other unique genomic clone, $\lambda P O D 3$, was subcloned and subjected to DNA sequence analysis. The entire gene has been sequenced including more than $500 \mathrm{bp}$ upstream from the start of transcription (Fig. 3). This lambda phage clone is $15 \mathrm{~kb}$ in length, and contains $1.5 \mathrm{~kb}$ of $5^{\prime}$-flanking sequences. Fig. 3 indicates the start of transcription, putative TATA box, and locations for putative cis-acting regulatory regions. Also shown is the location of a HinDIII site which was inserted via in vitro mutagenesis. This restriction will permit the joining of the peroxidase regulatory/promoter sequences to either the CAT or GUS reporter genes for fine structure mapping studies.

\section{SUMMARY}

In summary, we have determined that the alteration of the expression of the tobacco anionic peroxidase may lead to numerous phenotypic changes. Many of these phenotypes were unpredicted, and indicate that this enzyme has a number of critical functions in plant growth and development. The biochemical mechanisms which lead to these changes are within our reach, and it is felt that the results will not only 
shed light on the role of peroxidase in vivo, but will provide us with the means to better understand plant growth and the tools for plant improvement through genetic engineering.

\section{REFERENCES}

1. Lagrimini, L. M., Bradford, S. and Rothstein, S. Novel phenotypes in transgenic tobacco plants with reduced peroxidase activity. PLANTA (Manuscript submitted for publication).

2. Lagrimini, L. M. Genetically-altered peroxidase expression: Effect on cell growth and auxin metabolism. PLANTA (Manuscript submitted for publication).

3. Lagrimini, L. M. (1991). Altered phenotypes in plants transformed with chimeric tobacco peroxidase genes. In BIOCHEMICAL, MOLECULAR AND PHYSIOLOGICAL ASPECTS OF PLANT PEROXIDASES II, pp. 60-69, Eds. C. Penel, T. Gaspar and J. Lobarzewski, In Press.

4. Lagrimini, L. M. (1991). Wound-induced deposition of polyphenols in transgenic plants overexpressing peroxidase. PLANT PHYS. 96,577583.

5. Lagrimini, L. M., Bradford, S. and Rothstein, S. (1990). Peroxidaseinduced wilting in transgenic tobacco plants. THE PLANT CELL 2:7-18.

6. Rothstein, S. A. and Lagrimini, L. M. (1989). Silencing Gene Expression in Plants. In OXFORD SURVEYS OF PLANT MOLECULAR AND CELlular bIOLOGY, Ed. B. J. Miflin, Oxford University Press, Vol 6, pp. 55-78.

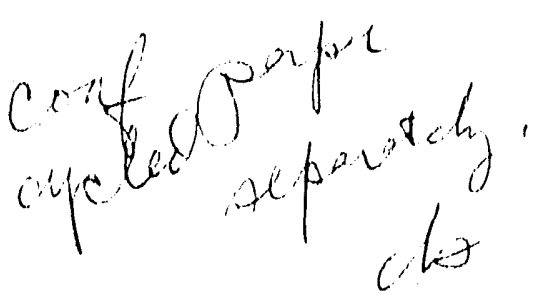



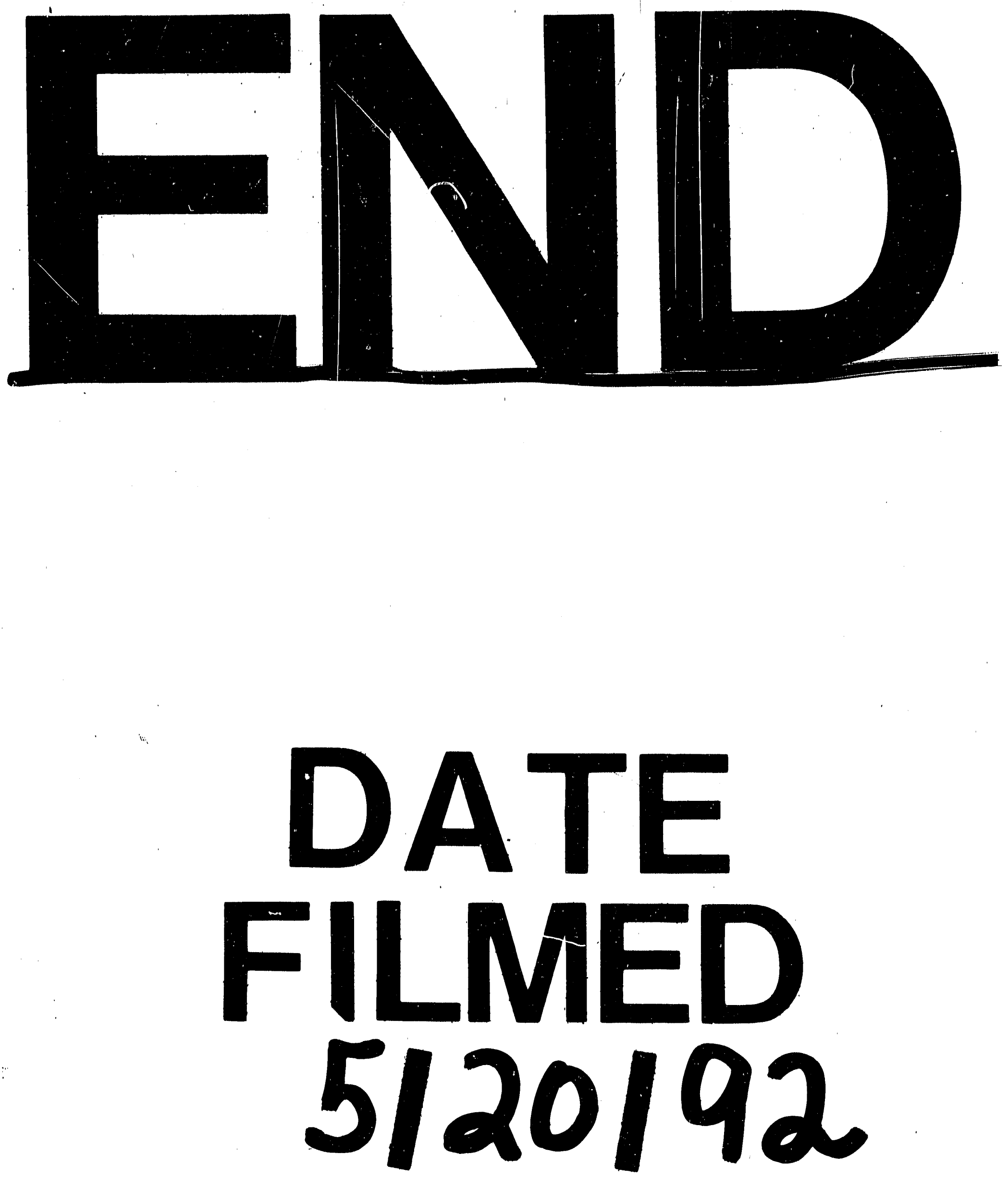
